\title{
Gestión de las reservas productivas en una PYME de Santo Domingo de los Tsáchilas
}

\author{
(Management of productive reserves in a SME of Santo \\ Domingo de los Tsáchilas)
}

\author{
Rodobaldo Martínez Vivar ${ }^{1}$, Alexander Sánchez Rodríguez ${ }^{1}$, Gelmar García Vidal ${ }^{1}$, \\ Reyner Pérez Campdesuñer ${ }^{1}$
}

\begin{abstract}
Resumen:
El desarrollo de una metodología para la organización del trabajo en la PYME, que posibilite la identificación de las reservas productivas existentes y con ello una consecuente mejora de los indicadores de productividad en el trabajo, constituye el objetivo de la presente investigación. Para el logro de este objetivo se propone un procedimiento adaptado a las condiciones del objeto de estudio práctico, el cual desde un enfoque holístico permitió la realización de los análisis que se desarrollan. Entre los principales resultados se pueden destacar los siguientes: la existencia de un desaprovechamiento de la jornada laboral de un $15 \%$ determinado fundamentalmente por pérdidas de tiempo eliminables, de igual manera se logró identificar la capacidad limitante del proceso de producción del medicamento natural Prostagerón, así como se identificaron problemas vinculados con el ruido, vibración, iluminación y ventilación, aunque no existen mayores inconvenientes que puedan perjudicar la salud de los trabajadores. Finalmente se logró incrementar la productividad del trabajo en un $35.42 \%$ respecto con el análisis desarrollado vinculado con la capacidad limitante detectada, al tiempo que en este primer análisis en cuanto a la observación de campo se puede indicar la posibilidad de un incremento productivo de un $327 \%$.
\end{abstract}

Palabras clave: Organización del trabajo, reservas productivas, productividad laboral, condiciones de trabajo.

\begin{abstract}
:
The development of a methodology for the organization of work in the SME, which enables the identification of existing productive reserves and thus a consequent improvement of the indicators of labor productivity is the goal of the present investigation. To achieve this objective a process adapted to the conditions of the object of practical study, which from a holistic approach allowed the realization of analysis for the proposed organization of work. The main results can be highlighted the following: the existence of a waste of working time $15 \%$ primarily determined by wasting time in the day, likewise it allowed identifying the limiting capacity of the production process of the natural medicine Prostageron, as well as problems associated with noise, vibration, lighting and ventilation were identified, although there are no major problems that could harm the health of workers. Finally he was able to increase labor productivity in $35.42 \%$ compared with the analysis developed linked to the detected limited capacity, while in this first analysis for field observation can indicate the possibility of a production increase of $327 \%$
\end{abstract}

Keywords: Work organization, productive reserves, labor productivity, working conditions.

\footnotetext{
${ }^{1}$ Universidad Tecnológica Equinoccial, Santo Domingo - Ecuador (\{rodobado.martinez, alexander.sanchez, germar.garcia, reyner.perez\} @ute.edu.ec).
} 


\section{Introducción}

La evolución del proceso de globalización, entre otros factores, ha distinguido el reconocimiento del talento humano $(\mathrm{TH})$ como un factor esencial para el logro de los resultados organizacionales y regionales, en época de críticas transformaciones estos recursos se distinguen como la ventaja competitiva de la contemporaneidad, (García Jarquín \& Aguilar Fernández, 2013; García Vidal, Sánchez Rodríguez, Martínez Vivar, \& Pérez Campdesuñer, 2016; Tapia \& Gómez Díaz, 2014; Zapata Rotundo \& Hernández, 2014).

El TH es el centro y fin del sistema de gestión de talento humano (GTH) (Castilla Sánchez, 2014; Cuesta Santos, 1990; Chiavenato, 2009; Fleitas, 2013), encargado de propiciar el desarrollo y consolidación de las competencias humanas, las que desde el siglo XX y con mayor énfasis en el XXI constituyen las reservas para impulsar el desarrollo (Arias Guevara \& Labrada Silva, 2008; Martínez Vivar, Marrero Fornaris, \& Pérez Campdesuñer, 2013; Sánchez Rodríguez, 2013) destacándose al TH como el fin y no el medio de este proceso (Arias Guevara \& Labrada Silva, 2008; Carrasco, 1999).

Bajo esta concepción gana espacio la organización del trabajo (OT), que constituye un proceso complejo, cuya meta esencial es el hombre, por lo cual, es preciso tener en cuenta las características propias de los segmentos de trabajadores y las condiciones para garantizar un clima laboral favorable, a partir de perfeccionar los métodos de la organización del trabajo individual y colectivo y con ello el incremento de la productividad en el trabajo.

Por su parte, la OT ha sido objeto de estudio de diversos autores (Agulló Tomás, 2011; Marsán Castellanos, Karell Rodríguez, Gutiérrez Rodríguez, Cuesta Santos, \& Moreno Rodríguez, 1999; Maynard, 2006; Meyers, 2000; Niebel \& Freivalds, 2009; Ramos Iglesias \& Bernal Rodríguez, 2012; Rivas Torres, 2011; Rocha Araújo, Correia Sampaio, Costa e Castro, Vieira Pinheiro, \& Macedo, 2014), quienes han desarrollado guías de acción para conducir estudios sobre esta temática, manifestándose de manera general un carácter marcadamente cuantitativo en los autores consultados, ofreciendo un amplio conjunto de técnicas para el desarrollo de este proceso.

El desarrollo socioeconómico de un país depende en gran medida de la administración de la producción y los servicios, por lo que constituye un reto lograr la máxima eficacia y eficiencia en este sentido. Ecuador en la actualidad no está exento de la anterior afirmación, destacándose la pertinencia de esta temática en diversos escenarios que han tratado de impulsar el desarrollo de la matriz productiva, visto este fenómeno no solo desde la estructuración macroeconómica del mercado (Hausmann, 2014; Parreño, 2014), sino desde una perspectiva microeconómica hacia lo que se tiene, donde se deben fomentar estudios que contribuyan con la identificación de las reservas productivas potenciales para favorecer los procesos y con ello lograr una mayor rentabilidad, originada desde la mejora en la estructuración de los costes de operaciones. 
Por tal razón se decidió realizar un estudio general de la productividad de la PYME, que busca contribuir al desarrollo de la matriz productiva. En un estudio previo desarrollado en esta PYME (Aceldo Rangel, 2015), se pueden observar un conjunto de insuficiencias asociadas con la OT, entre las que se pueden destacar:

- Desconocimiento del flujo de producción y su organización para el desempeño de los procesos.

- Déficits o Exceso de recursos humanos y (o) equipos para el desempeño de los procesos.

- Baja utilización de las capacidades instaladas.

- Limitaciones en la identificación de los indicadores de aprovechamiento de la jornada laboral.

Todo lo cual evidencia las limitaciones en la OT en la PYME objeto de estudio.

\section{La organización del trabajo. Conceptos y enfoques fundamentales}

La organización del trabajo es una disciplina científica que busca la optimización del uso de la capacidad laboral del hombre en el proceso de trabajo y la elevación de su calidad de vida laboral. Constituye un sistema donde los elementos fundamentales son: la cooperación y división del trabajo; los métodos; el servicio y organización del puesto de trabajo; la disciplina obrera; las condiciones laborales, la normación y la organización del salario.

Estos aspectos deben complementarse con los restantes elementos que generan en el trabajador la satisfacción laboral, entre los cuales se pueden argumentar: el contenido del trabajo; la estimulación moral y material; las relaciones entre subordinados y jefes; la participación; las posibilidades ascenso y la utilización del fondo de tiempo.

En el estudio del estado del arte, se evidencia una gran variedad de enfoques sobre OT (Garcia Remus \& Junco Campos, 2011; Jurado Jurado, 2015; Marsán Castellanos, et al., 1999; Maynard, 2006; Niebel \& Freivalds, 2009; Ormaza Murillo, Félix López, Real Pérez, \& Parra Ferié, 2015; Silva \& Navarro, 2012), quienes realizan sus aportes sobre la organización científica del trabajo; favoreciendo el punto de partida para los posteriores estudios sobre la temática. De igual modo destacan otros autores que si bien, en su generalidad, no estandarizan el término $\mathrm{TH}$, si orientan sus sobre la planificación del factor humano de forma general y su gestión en el nivel empresarial.

Para el desarrollo del análisis sobre los enfoques de OT, se construyó una matriz de datos, en función de las variables de OT identificadas por los autores consultados. Esta información se sometió a un análisis de Conglomerado Jerárquico. ${ }^{2}$ El dendrograma que se muestra en la Figura 1 , es el resultado del análisis con un corte en el nivel 5, muestra la presencia de dos grandes grupos: el primero recoge las propuestas de la última década del pasado siglo e inicios del presente, acen-

\footnotetext{
${ }^{2}$ En el análisis de clúster desarrollado se utilizó el método Ward, con la distancia euclidiana al cuadrado, según las características de la escala utilizada para valorar las variables en investigación. La validación de los resultados se desarrolló a través de la comparación de la misma muestra por el método no jerárquico
} 
tuando en sus aportes un enfoque más metodológico y filosófico que técnico, sin lograr la suficiente profundización en el cómo actuar, al tiempo que la profundidad de las variables tratadas varía entre ellos (Bustillo, 1994; Marsán Castellanos, et al., 1999; Ozaki, 2009; Puchol, 2012; Werther \& Davis, 2008). De igual manera se destacan autores (Marsán Castellanos, et al., 1999; Maynard, 2006; Niebel \& Freivalds, 2009) con un carácter marcadamente cuantitativo, ofreciendo un amplio conjunto de técnicas para el desarrollo de este proceso, obviándose los elementos cualitativos con una visión operativa que limita los análisis desde lo estratégico y su control.

El segundo grupo presenta propuestas más acercadas a la integración de los elementos evaluados (De Miguel Guzmán, 2006; Gonzáles Álvarez, 2012; Martínez Vivar, et al., 2013; Morales Cartaya, 2009; Sánchez Rodríguez, Martínez Martínez, \& Marrero Fornari, 2005), incorporando un detallado carácter metodológico e integral en sus propuestas (Nieves Julbe, 2006), de igual manera se destaca un adecuado enfoque de procesos para los análisis que se desarrollan, al tiempo que el enfoque sistémico y estratégico matizan como elementos fundamentales en sus propuestas.

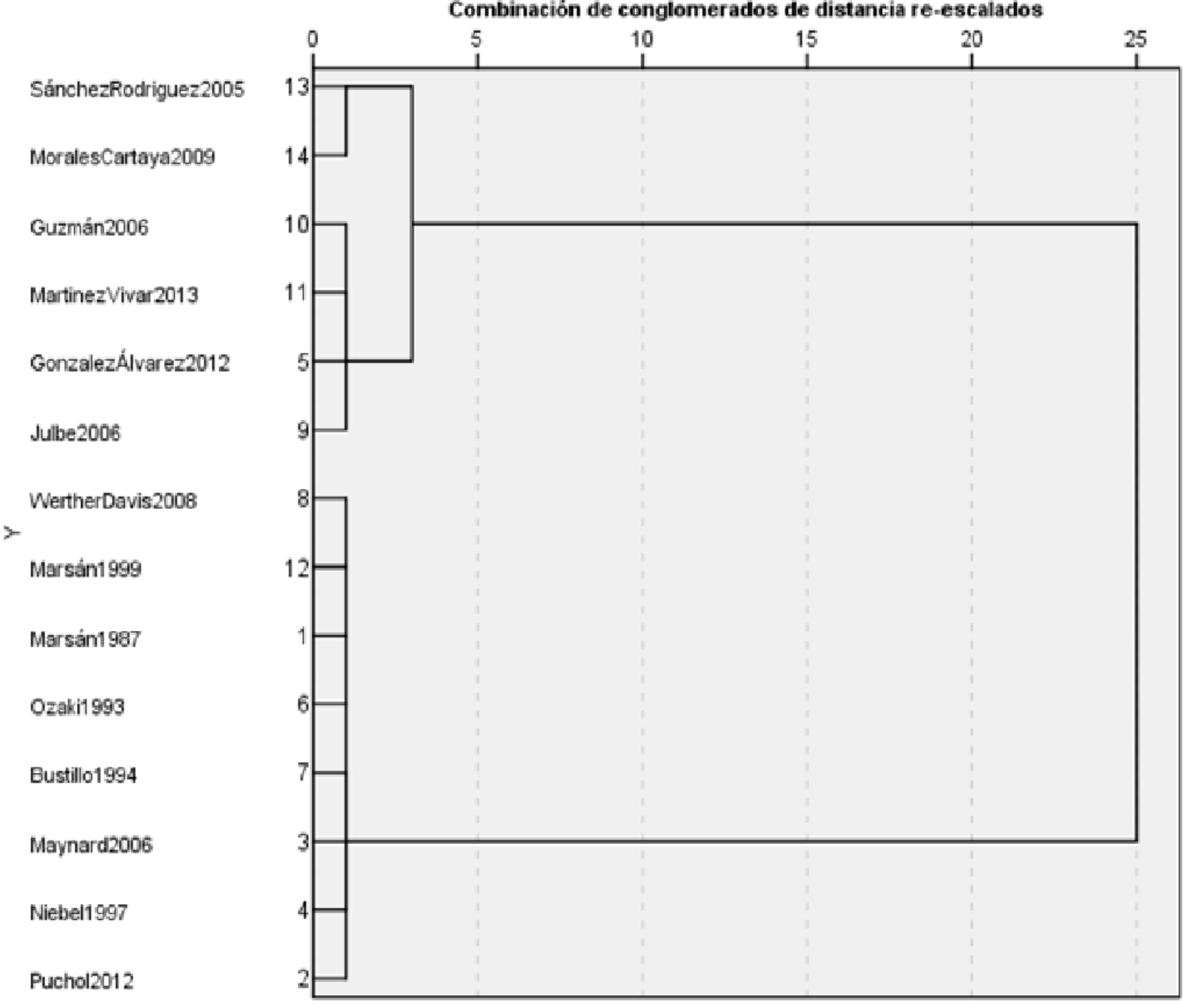

Dendrograma que utiliza una vinculación de Ward

Combinación de conglomerados de distancia réescalados

Figura 1. Dendrograma que utiliza una vinculación de Ward

Fuente: Elaboración propia en base a la información entregada por el SPSS 
Los elementos resultantes de los análisis desarrollados llevaron a los autores a tomar y adaptar, según las condiciones lo requieran el procedimiento propuesto por Nieves Julbe (2006) para el desarrollo de esta investigación, dado que este involucra de modo eficiente las variables analizadas como necesarias para el estudio que se pretende llevar a cabo.

\section{Metodología}

Para esta investigación se ha considerado como población y muestra el número total de colaboradores que prestan sus servicios en la Empresa, que ascienden a 19 trabajadores.

El procedimiento específico para desarrollar la organización del trabajo en la PYME se detalla a continuación:

Fase 1. Preparación inicial. Se lleva a cabo en tres pasos: Paso 1) Selección del equipo de trabajo; Paso 2) Capacitación del equipo de trabajo; Paso 3) Comunicar a los trabajadores el desarrollo del estudio.

Fase 2. Familiarización. Los pasos son: Paso 1) Caracterización de la organización; Paso 2) Caracterización del talento humano; Paso 3) Análisis de los procesos organizacionales.

Fase 3. Análisis de los elementos de organización del trabajo. Paso 1) Selección de o los procesos (u operaciones) a analizar; Paso 2). Descripción del proceso seleccionado; Paso 3) Diagnóstico de la OT del proceso seleccionado. Se realiza el estudio siguiendo el orden de los elementos del sistema de OT:

- División y cooperación del trabajo

- Métodos de trabajo

- Organización y servicio a los puestos de trabajo

- Medición y normación del trabajo

- Condiciones de trabajo: Humedad del aire y Temperatura; Contenido de polvo y de sustancias tóxicas en el ambiente; Nivel de ruido y de vibraciones; Nivel de iluminación; Condiciones estéticas del área; Condiciones de peligrosidad con que se desarrolla el trabajo; Disciplina laboral.

Fase 4. Ajustar las medidas técnico organizativas para la mejora de los elementos de OT en el proceso seleccionado. Paso 1) Identificación de las medidas técnico-organizativas; Paso 2) Implementación de las medida técnicos organizativas.

\section{Resultados}

Caracterización de la organización.- La empresa se dedica a la comercialización y producción de medicamentos naturales. Su actividad esencial está orientada hacia el consumo humano. Se elaboran y comercializan a nivel nacional 10 productos tal como se especifica en la Tabla 1 , los cuales cuentan con registro sanitario. 
La distribución del personal directo e indirecto hace referencia a la cantidad de colaboradores que participan en forma más cercana al proceso productivo, y una vez analizada la plantilla total el resultado es que cinco empleados son los que intervienen o forman parte del proceso productivo, mientras que el resto de la plantilla (15 colaboradores) son el personal indirecto de .

Tabla 1. Estadísticas de venta por producto

\begin{tabular}{|l|c|c|c|c|c|c|}
\hline \multirow{2}{*}{ Productos } & \multicolumn{2}{|c|}{ Diciembre-2014 } & \multicolumn{2}{c|}{ Noviembre-2014 } & \multicolumn{2}{c|}{ Octubre-2014 } \\
\cline { 2 - 7 } & Unidadad & Venta & Unidadad & Venta & Unidadad & Venta \\
\hline AllStres & 356 & $1.642,91$ & 604 & $2.708,82$ & 313 & $1.574,01$ \\
\hline $\begin{array}{l}\text { Amargo } \\
\text { Hepático }\end{array}$ & 433 & $2.047,45$ & 1094 & $4.950,48$ & 610 & $3.042,95$ \\
\hline Biobrón & 187 & 843,89 & 973 & $4.353,56$ & 433 & $2.135,81$ \\
\hline Dialsis & 141 & 845,71 & 403 & $2.442,20$ & 92 & 648,27 \\
\hline Diurex & 389 & $1.742,40$ & 998 & $4.515,70$ & 492 & $2.425,48$ \\
\hline Endobión & 466 & $4.429,86$ & 793 & $8.804,76$ & 403 & $5.162,30$ \\
\hline FactorU & 372 & $1.621,52$ & 465 & $2.065,53$ & 172 & 885,40 \\
\hline Glukonat & 234 & $1.041,40$ & 325 & $1.483,25$ & 413 & $2.039,32$ \\
\hline Herbalum & 352 & $2.073,38$ & 505 & $2.895,56$ & 302 & $1.912,14$ \\
\hline Prostagerón & 844 & $8.578,97$ & 1183 & $12.154,42$ & 1105 & $12.403,16$ \\
\hline \multicolumn{1}{|c|}{ Total } & $\mathbf{3 7 7 4}$ & $\mathbf{2 4 . 8 6 7 , 4 9}$ & $\mathbf{7 3 4 3}$ & $\mathbf{4 6 . 3 7 4 , 2 8}$ & $\mathbf{4 . 3 3 5 , 0 0}$ & $\mathbf{3 2 . 2 2 8 , 8 4}$ \\
\hline
\end{tabular}

Análisis de los procesos organizacionales.- Los procesos organizacionales se encuentran estructurados de la manera siguiente:

1. Procesos Estratégicos: Llevar a cabo las actividades de gestión de la dirección, para desarrollar, elaborar y comercializar medicamentos naturales, y satisfacer las necesidades de la población en la prevención y tratamiento de las diferentes patologías.

2. Procesos Operativos: Desarrollo de fórmulas de medicamentos naturales para diferentes tipos de patologías; Producción de medicamentos naturales bajo los estándares de calidad exigidos por el gobierno (Ley Orgánica de Salud No. 2006-67 y el Reglamento y Control de Productos Naturales de Uso Medicinal, según Acuerdo Ministerial 244) y recomendados por la norma ISO 9001; Una correcta gestión de comercialización de los medicamentos naturales elaborados.

3. Procesos de Apoyo: Gestión del personal altamente capacitado y motivado para la ejecución de los procesos y el cumplimiento de objetivos; Transparencia absoluta en el manejo de recursos financieros de la empresa, buscando la optimización de recursos y maximización de ganancias; Asegurar la continuidad de los procesos operativos, mediante la gestión documental, gestión de servicios generales, compras y contratación y mantenimiento de la infraestructura; Cumplir con las disposiciones legales vigentes a partir de una adecuada asesoría jurídica.

Análisis de los elementos de organización del trabajo.- Se decide por el grupo de expertos, cuáles procesos deben ser investigados. Se recomienda iniciar en los procesos claves ya que son los que determinan el cumplimiento de los objetivos organizacionales.

Tomando como base los niveles de ingresos que se detallan en la Tabla 1, se procede a realizar 
una priorización con el fin de poder determinar cuáles serán los productos que van a ser objeto de la investigación, dicho análisis es realizado en base al diagrama de Pareto, enfocando el estudio en el $80 \%$ de las ganancias que son generadas por el $20 \%$ de los productos elaborados.

Una vez realizado el diagrama de Pareto se pudo observar que el $20 \%$ de los productos que generan el $80 \%$ de los ingresos obtenidos son: Prostagerón, Endobión, Amargo Hepático y Diurex, es decir sobre los productos mencionados se centrará la investigación. Teniendo en cuenta estos resultados, la alta dirección de la organización ha decidido que el estudio de la investigación se centre prioritariamente en el producto Prostagerón.

Descripción del proceso seleccionado.- Se elaboró el diagrama OTIDA para representar el proceso de elaboración del producto escogido, en el diagrama se muestran las operaciones que se realizan, así como también los controles de calidad y demás actividades.

El proceso de producción inicia con la compra de $100 \mathrm{~kg}$ de materia prima (MP) destinados para la producción semestral, los que se dividen en: $30 \%$ de MP 1; 30\% de MP 2; $10 \%$ de MP 3; $10 \%$ de MP 4; $10 \%$ de MP 5; 10\% de MP 6. La MP para la elaboración de los productos que comercializa son plantas. Una vez recibida la MP se realiza un control de calidad para analizar las características organolépticas de las plantas que acaban de ingresar; esta actividad es realizada de manera manual y requiere de la labor de 1 operario. Luego siguen las siguientes actividades:

- Operación 1.- Mezclado de plantas.

- Operación 2.- Extracto hidroalcóholico.

- Operación 3.- Granulado- control de procesos.

- Operación 4.- Secado.

- Operación 5.- Encapsulado.

- Operación 6.- Envasado y sellado.

- Operación 7.- Estuchado y etiquetado.

- Operación 8.- Loteado.

- Operación 9.- Embalaje final.

- Transporte a bodega

- Almacenamiento del producto terminado.

Diagnóstico de la organización del trabajo del proceso seleccionado.- Para desarrollar el diagnóstico se utilizó la técnica del balance de carga capacidad. Una vez identificada la capacidad limitante se realizarán las sugerencias necesarias para contribuir con: la mejora de la cooperación y división del trabajo, la organización y servicio a los puestos de trabajo, las condiciones de trabajo y los métodos de trabajo en las restricciones en estudio.

Seguidamente se desarrolla un análisis de aprovechamiento de la jornada laboral (AJL) para el proceso de fabricación del medicamento natural Prostagerón. El análisis se realiza a través de la técnica de observación continua del muestreo de observaciones instantáneas (MOI), para ello se 
utiliza el software profesional MEDTRAB. Los principales resultados, luego de la aplicación de la herramienta, se muestran en las tablas 2 y 3.

Tabla 2. Resumen del Modelo del MOI

\begin{tabular}{|c|c|c|c|}
\hline Días & Tiempos Normables & Tiempos No Normables & Observaciones \\
\hline 1 & 72 & 28 & 100 \\
\hline 2 & 177 & 23 & 200 \\
\hline 3 & 175 & 30 & 205 \\
\hline
\end{tabular}

Tabla 3. Recalculo de observaciones

\begin{tabular}{|c|c|c|c|c|c|c|}
\hline Días & $\begin{array}{c}\mathbf{P} \text { (veces } \\
\text { trabajando) }\end{array}$ & $\begin{array}{c}\mathbf{N} \text { (total de obser- } \\
\text { vaciones) }\end{array}$ & $\begin{array}{c}\text { Paj (Fraccín de } \\
\text { veces trabajando) }\end{array}$ & $\begin{array}{c}\text { Naj(acumulado de } \\
\text { observaciones) }\end{array}$ & $\begin{array}{c}\text { paj (acumulado veces } \\
\text { trabajando) }\end{array}$ & $\begin{array}{c}\mathbf{N d j} \text { (total de observa- } \\
\text { ciones necesarias) }\end{array}$ \\
\hline 1 & 72 & 100 & 0,72 & 100 & 0,72 & 622,22 \\
\hline 2 & 177 & 200 & 0,89 & 300 & 0,83 & 327,71 \\
\hline 3 & 175 & 205 & $\mathbf{0 , 8 5}$ & 505 & 0,84 & 304,76 \\
\hline
\end{tabular}

Como el Naj > Ndj se detiene el MOI el día 3

Como puede consultarse, luego de aplicadas las herramientas puede concluirse que existe un AJL del $85 \%$, el cual se determinó para un nivel de significancia del 0.039 determinándose como válido para inferir los resultados que se plantean. Las principales pérdidas de tiempo detectadas se analizarán más adelante, según se muestra en la Tabla 4.

Análisis de carga capacidad para el proceso de fabricación de Prostagerón.- De acuerdo con los cálculos realizados se concluye que la demanda dependiente para el almacenamiento 1 es de: $82.60 \mathrm{~kg}$ de MP 1, 82.60kg de MP 2, 27.53kg de MP 3, 27.53kg de MP 4, 27.53kg de MP 5, $27.53 \mathrm{~kg}$ de MP 6 , además la operación 5 se encuentra en un balance de acuerdo con la identificación de las capacidades limitantes, mientras que las demás operaciones están siendo subutilizadas de acuerdo con la capacidad total calculada, teniendo como resultado final que el almacenamiento 2 tiene una producción total de $234.22 \mathrm{~kg} / \mathrm{m}$, o sea 7807 frascos de Prostagerón al mes.

$$
C L=C T_{5}=Q_{5}=239 \mathrm{~kg} / \mathrm{mes}
$$

A continuación se realiza un análisis basado en la producción actual y la producción obtenida bajo el criterio de capacidad limitante, que permite conocer los ingresos posibles una vez ajustada la capacidad limitante. Para el análisis de los indicadores de productividad del trabajo se utiliza el método de cálculo natural desde un análisis social.

Existe una dinámica productiva al ajuste de la capacidad de producción que es de $327 \%$, lo cual determina que se podría triplicar la producción actual ajustándose a la capacidad limitante encontrada durante la investigación realizada. Al analizar la dinámica productiva se puede evidenciar que no se está aprovechando al máximo las capacidades instaladas dentro la empresa. Cabe señalar que para el producto en análisis existe según el área de comercialización de la empresa una demanda insatisfecha de 10261 unidades al año, lo que determina un total aproximado de 855 
unidades al mes, observándose la posibilidad del proceso productivo para cubrir estas necesidades

Tabla 4. Determinación de las capacidades productivas

\begin{tabular}{|c|c|c|c|c|}
\hline OP & $\begin{array}{c}\text { Fórmula } \\
\boldsymbol{C u}=\frac{\boldsymbol{K g}}{\boldsymbol{h}} * \frac{\boldsymbol{h}}{\boldsymbol{d}} * \frac{\boldsymbol{d}}{\boldsymbol{m}} * \boldsymbol{A J \boldsymbol { L }}\end{array}$ & $\begin{array}{c}\text { Fúrmula } \\
\boldsymbol{C T}=\boldsymbol{C u} * \boldsymbol{N T} / \mathrm{mes}\end{array}$ & $\begin{array}{c}\mathrm{CT} \text { - } \\
\mathrm{Kg} / \mathrm{mes}\end{array}$ \\
\hline 1 & $C u_{o p 1}=\frac{20 \mathrm{Kg}}{6 h} * \frac{7,5 h}{d} * \frac{20 d}{m} * 0,85$ & 425 & $C T_{o p 1}=425 * 1$ & 425 \\
\hline 2 & $C u_{o p 2}=\frac{5 \mathrm{Kg}}{0.75 h} * \frac{7,5 h}{d} * \frac{20 d}{m} * 0,85$ & 850 & $C T_{o p 2}=850 * 1$ & 850 \\
\hline 3 & $C u_{o p 3}=\frac{15 K g}{5.75 h} * \frac{7,5 h}{d} * \frac{20 d}{m} * 0,85$ & 333 & $C T_{o p 3}=333 * 1$ & 333 \\
\hline 4 & $C u_{o p 4}=\frac{15 K g}{2 h} * \frac{7,5 h}{d} * \frac{20 d}{m} * 0,85$ & 956 & $C T_{o p 4}=956.25 * 1$ & 956 \\
\hline 5 & $C u_{o p 5}=\frac{7.5 \mathrm{Kg}}{8 h} * \frac{7,5 h}{d} * \frac{20 d}{m} * 0,85$ & 119.5 & $C T_{o p 5}=119.5 * 2$ & 239 \\
\hline 6 & $C u_{o p 6}=\frac{7.5 \mathrm{Kg}}{4 h} * \frac{7,5 h}{d} * \frac{20 d}{m} * 0,85$ & 239 & $C T_{o p 6}=239 * 2$ & 478 \\
\hline 7 & $C u_{o p 7}=\frac{7.5 \mathrm{Kg}}{7.50 h} * \frac{7,5 h}{d} * \frac{20 d}{m} * 0,85$ & 127.5 & $C T_{o p 7}=127.5 * 2$ & 255 \\
\hline 8 & $C u_{o p 8}=\frac{15 \mathrm{Kg}}{2 h} * \frac{7,5 h}{d} * \frac{20 d}{m} * 0,85$ & 956 & $C T_{o p 8}=956 * 1$ & 956 \\
\hline 9 & $C u_{o p 9}=\frac{15 \mathrm{Kg}}{0.50 h} * \frac{7,5 h}{d} * \frac{20 d}{m} * 0,85$ & 3825 & $C T_{o p 9}=3835 * 1$ & 3825 \\
\hline
\end{tabular}

Leyenda: $\mathrm{Cu}=$ Capacidad Unitaria, $\mathrm{CT}=$ Capacidad Total, OP = Operación, NT = Número de trabajadores, $\mathrm{AJL}=$ Aprovechamiento de la jornada laboral, $\mathrm{h}=$ horas, $\mathrm{d}=$ días, $\mathrm{m}=$ mes.

Tabla 5. Ingresos por producción

\begin{tabular}{|c|c|c|c|c|c|}
\hline $\begin{array}{c}\text { Producción actual } \\
\text { (Unidades) }\end{array}$ & Precio \$ & Ingresos & $\begin{array}{c}\text { Producción (Ajuste } \\
\text { CL unidades) }\end{array}$ & Precio \$ & Ingresos \\
\hline 1044 & 14,75 & 15399,00 & 7807 & 14,75 & 115153,25 \\
\hline
\end{tabular}

Tabla 6. Dinámica productiva

\begin{tabular}{|c|c|c|}
\hline $\begin{array}{c}\text { Productividad 1 } \\
P T_{1}=\frac{V p}{N T}\end{array}$ & $\begin{array}{c}\text { Productividad 2 } \\
P T_{2}=\frac{V p}{N T}\end{array}$ & $\begin{array}{c}\text { Dinámica de PT } \\
\text { NT }\end{array}$ \\
\hline 261 unidades $\mathrm{F} / \mathrm{m}-\mathrm{t}$ & 1115 unidades $\mathrm{F} / \mathrm{m}-\mathrm{t}$ & $327 \%$ \\
\hline
\end{tabular}

División y cooperación del trabajo.- Para la correcta realización de los procesos que se realizan en la empresa y con el objetivo de mejorar la eficiencia conjunta de los trabajadores, se llevan a cabo diferentes técnicas de división y cooperación del trabajo para lograr identificar y explotar las reservas productivas, incrementando la productividad en el trabajo.

De acuerdo con lo expuesto en la Tabla 7, se ha considerado que para la OP1 solo se requiere la presencia de un trabajador, que se encargará de realizar también la OP2, por lo que en esta última operación se ha decidido no ubicar otro trabajador. En la OP3 se ha calculado que es necesa- 
rio un operario, que igualmente será el encargado de ejecutar la OP4; mientras que para la OP5 se requieren dos operarios para poder concluir con la tarea en un tiempo menor y con mayor eficiencia; en la OP6 se necesita un operario, al igual que en las OP7 y OP8; y la OP9 es realizada por el mismo trabajador que ejecuta la OP8. Por lo tanto, se constata que para llevar a cabo la producción del Prostagerón es necesario incrementar el número de operarios que intervienen en este proceso en tres nuevos trabajadores, es decir se requieren siete operarios con el fin de maximizar la producción y obtener mayores beneficios económicos. En lo que se refiere a los equipos se ha optado por mantener los mismos con los que la empresa cuenta actualmente debido a que su uso durante la producción es subutilizado.

Tabla 7. Determinación de número de trabajadores y equipos

\begin{tabular}{|c|c|c|c|c|c|c|c|c|c|}
\hline OP & $\mathbf{Q}$ & $\mathbf{C u}$ & $\mathbf{C T}$ & $\begin{array}{c}\% \text { Utili- } \\
\text { zación }\end{array}$ & $\mathbf{N T}$ & $\begin{array}{c}\mathbf{N T} \\
\text { actuales }\end{array}$ & $\begin{array}{c}\mathbf{N T} \\
\text { decididos }\end{array}$ & $\begin{array}{c}\text { Equipos } \\
\text { actuales }\end{array}$ & $\begin{array}{c}\text { Equipos } \\
\text { decididos }\end{array}$ \\
\hline 1 & 275,33 & 425 & 425 & 64,78 & 0,65 & 0 & 0 & 0 & 0 \\
\hline 2 & 275,33 & 850 & 850 & 32,39 & 0,32 & 1 & 1 & 2 & 2 \\
\hline 3 & 275,33 & 333 & 333 & 82,68 & 0,83 & 1 & 1 & 1 & 1 \\
\hline 4 & 229,44 & 956 & 956 & 24 & 0,24 & 0 & 0 & 0 & 0 \\
\hline 5 & 239 & 119,5 & 239 & 100 & 2 & 2 & 2 & 0 & 0 \\
\hline 6 & 234,22 & 239 & 478 & 49 & 0,98 & 0 & 1 & 1 & 1 \\
\hline 7 & 234,22 & 127,5 & 255 & 91,85 & 1,84 & 0 & 1 & 0 & 0 \\
\hline 8 & 234,22 & 956 & 956 & 24,5 & 0,25 & 0 & 1 & 1 & 1 \\
\hline 9 & 234,22 & 3825 & 3825 & 6,12 & 0,06 & 0 & 0 & 0 & 0 \\
\hline
\end{tabular}

$\mathrm{OP}=$ Operación, $\mathrm{Q}=$ Capacidad, $\mathrm{Cu}=$ Capacidad Unitaria, $\mathrm{CT}=$ Capacidad Total,

NT= Número de trabajadores.

Métodos de trabajo.- Se analiza el proceso de encapsulado del producto escogido para el estudio, por haber resultado la capacidad limitante del sistema, el cual tiene una duración de 8 horas por cada 60000 cápsulas, trabajo que es realizado por dos operarios, por lo cual el tiempo estimado de producción es de 96 segundos por cada 100 cápsulas de Prostagerón. Para lo cual se desarrolla la herramienta del diagrama hombre máquina, que explica los tiempos para la realización de la operación analizada.



Figura 2. Diagrama Hombre-Máquina

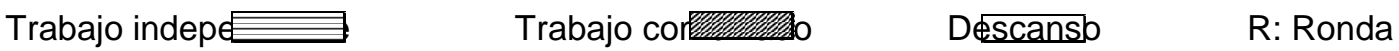

El número de rondas que se efectúan durante la $\mathrm{JL}$ es de $271 \mathrm{R} / \mathrm{JL}$, esto equivale al número de lotes producidos en el día por cada operario. El volumen de producción equivalente de los lotes elaborados por los operarios es de 451 frascos durante toda la JL, que multiplicando por el número de días que se laboran al mes da un resultado de $270,60 \mathrm{~kg} / \mathrm{mes}$, que transformando a frascos 
de productos se obtiene como resultado 9020.

Con la valoración del método registrado contra los datos recopilados en la información de campo sobre la capacidad de esta operación se puede concluir que:

Tabla 8. Ingresos por producción

\begin{tabular}{|c|c|c|c|c|c|c|}
\hline $\begin{array}{c}\text { Producción } \\
\text { actual (u) }\end{array}$ & Precio $\$$ & Ingresos & $\begin{array}{r}\text { Prod } \\
\text { (ajuste }\end{array}$ & & Precio $\$$ & Ingresos \\
\hline 1044 & 14,75 & 15399,00 & & & 14,75 & 133045 \\
\hline \multicolumn{7}{|c|}{ Tabla 9. Dinámica productiva } \\
\hline \multicolumn{2}{|c|}{$\begin{array}{l}\text { Productividad } 1 \\
\qquad P T_{1}=\frac{V p}{N T}\end{array}$} & \multicolumn{2}{|c|}{$\begin{array}{c}\text { Productividad } 2 \\
P T_{2}=\frac{V p}{N T}\end{array}$} & \multicolumn{3}{|c|}{$\begin{array}{c}\text { Dinámica de PT } \\
T=\frac{P T 2-P T 1}{P T 1} * 100\end{array}$} \\
\hline \multicolumn{2}{|c|}{261 unidades F/m-t } & \multicolumn{2}{|c|}{1288 unidades $\mathrm{F} / \mathrm{m}-\mathrm{t}$} & \multicolumn{3}{|c|}{$393 \%$} \\
\hline
\end{tabular}

Una vez utilizada la herramienta del diagrama hombre-máquina se determinó que existe una dinámica productiva de 393\%, lo cual determina que se podría triplicar la producción. Por ende los datos iniciales no responden totalmente a las verdaderas condiciones de desarrollo de esta actividad. Observándose reservas productivas importantes para su ejecución.

Organización y servicio a los puestos de trabajo.- Al estudiar el servicio de los puestos de trabajo se pudo evidenciar que el área de producción cuenta con las mayores facilidades para la realización de su respectivo trabajo a fin de que este no sea interrumpido de ninguna manera contando con amplios stocks de materia prima que va ser utilizada en el proceso productivo.

Condiciones de trabajo.- Para conocer las condiciones de trabajo en la que los trabajadores laboran se decidió aplicar una encuesta a todos los colaboradores de la organización, dicha encuesta fue procesada con el IBM SPSS Statistics. Para el desarrollo de este procesamiento se utilizó el paquete estadístico SPSS versión 16.0, con el fin de confirmar la fiabilidad y validez del instrumento diseñado con un coeficiente de Alpha de Cronbach de 0,861 y un coeficiente de Kaiser- Meyer- Olkin de 0,814.

Según las preguntas correspondientes a la dimensión de ruido se pudo evidenciar que el ruido en el ambiente de trabajo no produce molestias, por lo cual el trabajo no se ve interrumpido por este elemento, sin embargo se pudo constatar que según las respuestas no se han realizado mediciones acerca del nivel del ruido producido en los puestos de trabajo para determinar si fuese necesario la utilización de protección o en su caso plantear medias de prevención de tipo auditivo.

Al analizar la dimensión acerca de la vibración, los encuestados expresaron que la empresa no dispone de maquinaria o herramientas que produzcan dicho efecto nocivo, sin embargo dispone de un programa de mantenimiento y prevención a fin de que se encuentre en óptimas condiciones, cabe recalcar que la empresa no ha realizado mediciones acerca del nivel de vibración con un especialista en el campo. 
Disciplina laboral.- Dentro del proceso productivo existe un régimen estricto en cuanto al cumplimiento de los horarios, cabe recalcar que la principal indisciplina es la de conversar entre compañeros en horarios de trabajo, lo cual provoca desconcentración e incluso la disminución de la productividad. Mientras que en el área administrativa se destaca un mayor número de actividades innecesarias que inciden en el AJL, como: excesivo uso del teléfono celular, uso inadecuado del computador, abuso en cuanto a los permisos en horarios de oficina, interrupción de las actividades por ir a retirar impresiones en otra oficina, exceso de dialogo entre compañeros de temas inherentes a la empresa.

Ajuste de las medidas técnico organizativas para la mejora de los elementos de OT en el proceso seleccionado.- Se establecieron las medidas organizativas para la explotación de las reservas existentes, quedando elaborado el plan de perfeccionamiento de la OT.

Para erradicar la indisciplina laboral se plantean varias medidas con las cuales se pretende elevar el AJL para explotar el 15\% de reserva e incrementar el aprovechamiento de la jornada laboral al $100 \%$, entre dichas medidas se mencionan:

- No se recibirán visitas en horas laborables de personas ajenas a los procesos de la organización, salvo casos de fuerza mayor y en cuyo caso deberán recibirlas en lugares destinados a ese fin con el conocimiento y autorización del patrono o sus representantes.

- No permanecer en áreas de acceso restringido, interrumpiendo su labor o la de sus compañeros de trabajo.

- Los trabajadores no podrán interrumpir sus labores contestando su teléfono celular, en caso de portarlo deberán tenerlo apagado durante las horas de labores y de atención al público, tampoco podrán comer o ingerir bebidas durante ese tiempo.

Mejora de las condiciones de trabajo.- Dentro de las causas que afectan el clima organizacional se destacan: Déficit de personal, inseguridad laboral, sobrecarga laboral, deficiente climatización; para ello se han establecido algunas propuestas de mejora, como:

- Realizar mediciones del ruido y vibraciones en cada puesto de trabajo.

- Contar con protectores antideslumbrantes para evitar molestias ocasionadas por la luz.

- Realizar un mantenimiento preventivo planificado a instalaciones, mobiliario y equipos.

- Analizar si la distribución de los espacios, la ubicación (situación) de las personas, el mobiliario, etc., es la apropiada para la realización del trabajo.

De esta manera se alcanzará el 100\% del AJL, lo que permitirá conocer las nuevas capacidades totales en cada operación del proceso.

$$
C L=C T_{5}=Q_{5}=281 \mathrm{~kg} / \mathrm{mes}
$$


Tabla 10. Cálculo de los ingresos

\begin{tabular}{|c|c|c|c|c|c|}
\hline $\begin{array}{c}\text { Producción } \\
\text { actual (Uni) }\end{array}$ & Precio \$ & Ingresos & $\begin{array}{c}\text { Producción } \\
\text { (ajuste CL- Uni) }\end{array}$ & Precio \$ & Ingresos \\
\hline 1044 & 14,75 & 15399,00 & 10574 & 14,75 & 155966.50 \\
\hline
\end{tabular}

Tabla 11. Cálculo de la productividad

\begin{tabular}{|c|c|c|}
\hline $\begin{array}{c}\text { Productividad 2 } \\
P T_{2}=\frac{V p}{N T}\end{array}$ & $\begin{array}{c}\text { Productividad 3 } \\
P T_{3}=\frac{V p}{N T}\end{array}$ & $\Delta P T=\frac{P T 3-P T 2}{P T 2} * 100$ \\
\hline 1115 unidades F/m-t & 1510 unidades F/m-t & $35.42 \%$ \\
\hline
\end{tabular}

Es notorio que al mejorar el AJL al 100\% la productividad aumenta en 1510 unidades, reflejando un incremento del $35.42 \%$ en relación con la productividad obtenida cuando no se estaba aprovechando por completo la jornada laboral.

\section{Conclusiones}

- Durante la investigación se pudo determinar que existe gran diversidad de enfoques en lo referente a la organización de trabajo, razón por la cual se escogió un enfoque de carácter metodológico e integral que enfatiza el análisis de procesos.

- Se desarrolló un procedimiento que consistió en cuatro fases, que parten desde la selección y preparación del equipo de trabajo, caracterización de la organización con su talento humano y procesos, para continuar con el análisis de los elementos de la organización y presentar un diagnóstico de manera que se pueda establecer un conjunto de medidas técnico organizativas para el mejoramiento de la OT.

- El aprovechamiento de la jornada laboral dentro del proceso de producción no está siendo explotado al máximo, teniendo un $15 \%$ de tiempo de interrupciones, que disminuye la capacidad total de producción. De igual manera existen reservas productivas para incrementar el régimen de rendimiento de la operación 5 (Encapsulado), que se considera como limitante.

- Al estudiar las dimensiones referentes a la organización de trabajo se puede concluir que en la empresa existe una buena organización en cuanto al trabajo que realiza cada colaborador dentro de la empresa, en relación al ruido, vibración, iluminación y ventilación se determinó que no existen mayores inconvenientes que puedan perjudicar la salud de los trabajadores.

- Los resultados obtenidos permiten plantear la posibilidad de incrementar la productividad del trabajo en un $35.42 \%$ respecto con el análisis desarrollado vinculado con la capacidad limitante detectada, al tiempo que en este primer análisis respecto a la observación de campo se puede indicar la posibilidad de un incremento productivo de un $327 \%$. 


\section{Bibliografía}

Aceldo Rangel, J. A. (2015). Introducción del enfoque de competencias laborales en la gestión de talento humano del Laboratorio Fitofarmacéutico, de Santo Domingo de los Tsáchilas. Unpublished Tesis en opción al título de Ingeniero de Empresas y Negocios, Universidad Tecnológica Equinocial, Santo Domingo de los Tsáchilas.

Agulló Tomás, E. (2011). Nuevas formas de organización del trabajo y la empleabilidad. Oviedo: Universidad de Oviedo.

Arias Guevara, M. d. I. Á., \& Labrada Silva, C. (2008). Estudios sobre desarrollo local innovación social y género. La Habana: Editorial Academia.

Bustillo, C. (1994). La gestión de recursos humanos y la motivación de las personas. Revista Capital Humano(73), 17-28.

Carrasco, C. (1999). Mujeres y economía. Nuevas perspectivas para viejos y nuevos problemas. Barcelona: Icaria-Antrazyt.

Castilla Sánchez, A. (2014). Optimización del talento en las organizaciones: una mirada desde la realidad de la Dirección de $\mathrm{RRHH}$. Athenea Digital (Revista de Pensamiento e Investigación Social), 14(2), 285-291.

Cuesta Santos, A. (1990). Organización del Trabajo y Psicología Social. La Habana: Ciencias Sociales.

Chiavenato, I. (2009). Gestión del talento humano. (Tercera ed.). México, D.F.: McGRAWHILL/INTERAMERICANA EDITORES, S.A. DE C.V.

De Miguel Guzmán, M. (2006). Tecnología para la planeación integral de los recursos humanos. Aplicación en entidades hoteleras del destino Holguín. Unpublished Tesis en opción al grado científico de doctor en ciencias técnica, Instituto Superior Politécnico Jose Antonio Echeverría, Holguín.

Fleitas, S. (2013). La gestión del talento humano y del conocimiento. Revista Latinoamericana de Psicologí, 45(1), 157-160.

García Jarquín, B., \& Aguilar Fernández, M. (2013). El modelo Mondragón desde la perspectiva de los determinantes de la ventaja competitiva nacional. Gestión y Estrategia(44), 107-123.

Garcia Remus, M., \& Junco Campos, A. M. (2011). Cómo abordar la organización del trabajo en entidades de regulación y control. Transporte Desarrollo y Medio Ambiente, 31(2/3), 37-43.

García Vidal, G., Sánchez Rodríguez, A., Martínez Vivar, R., \& Pérez Campdesuñer, R. (2016). Estudio sobre los emprendimientos de la economía simple en el Ecuador. CienciasHolguín, 22(1), 1-16. 
Gonzáles Álvarez, R. (2012). Diseño de un procedimiento para realizar el autocontrol del sistema de gestión integrado de capital humano. Ingeniería Industrial, 33(1), 41-49.

Hausmann, R. (2014). La economía vista desde el cerebro. Retrieved 8 enero, 2015, from http://www.project-syndicate.org/commentary/ricardo-hausmann-proposes-an-alternativeapproach-to-economic-development-based-on-how-the-human-brain-functions/spanish

Jurado Jurado, J. C. (2015). Hacia una arqueología de la administración. Cuadernos de Administración, 28(50), 1-24.

Marsán Castellanos, J., Karell Rodríguez, A., Gutiérrez Rodríguez, M., Cuesta Santos, A., \& Moreno Rodríguez, O. (1999). La organización del trabajo. (Vol. I). México: Instituto Politecnico Nacional.

Martínez Vivar, R., Marrero Fornaris, C. E., \& Pérez Campdesuñer, R. (2013). Variables vinculadas con la planeación de recursos humanos a nivel territorial. Ingeniería Industrial, $4(2), 120-129$.

Maynard, H. (2006). Manual del ingeniero industrial (5ta ed.). México: McGraw-Hill / Interamericana de México

Meyers, F. E. (2000). Estudios de tiempos y movimientos. Para la manufactura ágil. (2da ed.). México: Pearson Educación.

Morales Cartaya, A. (2009). Capital Humano. Hacia un sistema de gestión en la empresa cubana. La Habana: Editora Política.

Niebel, B. W., \& Freivalds, A. (2009). Ingeniería industrial: Métodos, estándares y diseño del trabajo. (12ma ed.). México, D.F.: McGRAW-HILL/INTERAMERICANA EDITORES, S.A. DE C.V.

Nieves Julbe, A. (2006). Procedimiento de aplicación de la correlación estadística para la determinación de las necesidades de personal en entidades hoteleras. Unpublished Tesis para optar por el Título de Master, Universidad de Holguín" Oscar Lucero Moya".

Ormaza Murillo, M. P., Félix López, M., Real Pérez, G. L., \& Parra Ferié, C. (2015). Procedimiento para el diagnóstico de la organización del trabajo en la carrera agroindustrias. Ingenieria Industrial, 36(2), 126-137.

Ozaki, M. (Ed.). (2009). Cambios tecnológicos y relaciones laborales (Reimpresión ed.). Madrid: Ministerio de trabajo y Seguridad Social.

Parreño, L. (Ed.). (2014). Reporte de Consistencia Macroeconómica (RCM). Quito: Pontifica Universidad Católica del Ecuador.

Puchol, L. (2012). Dirección y gestión de recursos humanos (7ma ed.). Madrid: Ediciones Díaz de Santos, S.A. 
Ramos Iglesias, L., \& Bernal Rodríguez, J. (2012). Procedimiento para el estudio de la organización del trabajo en empresas cubanas. Revista Avanzada Científica, 15(1), 63-69.

Rivas Torres, F. E. (2011). Procesos y Organización del Trabajo: Reflexión desde una perspectiva social. Gaceta Laboral, 17(3), 309-323.

Rocha Araújo, D., Correia Sampaio, F. M., Costa e Castro, M., Vieira Pinheiro, S. A., \& Macedo, A. P. (2014). Teste no tempo: da teoria clássica da administração à organização atual do trabalho de Enfermagem. Revista de Enfermagem Referência, 4(2), 111-120.

Sánchez Rodríguez, A. (2013). Capital Humano, relaciones laborales y gestión por competencias en el proceso de cambio empresarial en Cuba: de 1989 a 2011. Unpublished Tesis en opción al grado de Doctor en Ciencias Económicas, Universidad de Valladolid, Valladolid.

Sánchez Rodríguez, A., Martínez Martínez, C. C., \& Marrero Fornari, C. E. (2005). Como gestionar los recursos humanos sobre la base de competencias laborales. Ingeniería Industrial, 26(2), 3-10.

Silva, J. L., \& Navarro, V. L. (2012). Work organization and the health of bank employees. Revista Latino-Americana de Enfermagem (RLAE), 20(2), 226-234.

Tapia, R. A., \& Gómez Díaz, M. d. R. (2014). Estrategias de gestión del conocimiento para generar ventajas competitivas en pequeñas y medianas empresas en el estado de México. Gestión y Estrategia(46), 55-71.

Werther, W. B., \& Davis, K. (2008). Administración de recursos humanos. El capital humano de las empresas (Sexta ed.). México, D.F.: McGRAW-HILL/INTERAMERICANA EDITORES, S.A. de C.V.

Zapata Rotundo, G. J., \& Hernández, A. (2014). Origen de los recursos y ventajas competitivas de las organizaciones: reflexiones teóricas. Utopia y Praxis Latinoamericana, 19(64), 735-759. 